\title{
Structured frameworks to increase the transparency of the assessment of benefits and risks of medicines: Current status and possible future directions
}

Francesco Pignatti (1), Deborah Ashby (2), Eric P. Brass (3), Hans-Georg Eichler (1), Patrick Frey (4), Hans Hillege (5), Akiko Hori (6), Bennett Levitan (7), Lawrence Liberti (8), Ragnar E. Löfstedt (9), Neil McAuslane (8), Alain Micaleff (10), Rebecca A Noel (11), Douwe Postmus (1), Ortwin Renn (12), Barbara J Sabourin (13), Tomas Salmonson (14), Stuart Walker (8).

(1) The European Medicines Agency, London, United Kingdom.

(2) School of Public Health, Imperial College London, London, United Kingdom.

(3) Department of Medicine, Harbor-UCLA Medical Center, Torrance, California, U. S. A.

(4) Food and Drug Administration, Silver Spring, Maryland, U. S. A.

(5) University of Groningen, The Netherlands.

(6) Pharmaceuticals and Medical Devices Agency, Tokyo, Japan.

(7) Janssen Pharmaceutical research and Development, Titusville, New Jersey, U. S. A.

(8) Centre for Innovation in Regulatory Science (CIRS), London, United Kingdom.

(9) Risk Research Group, Centre for Environmental Strategy, University of Surrey, Guildford, United Kingdom

(10) Merck Serono, Geneva, Switzerland.

(11) Eli Lilly and Company, Indianapolis, Indiana, U. S. A.

(12) Institut für Sozialwissenschaften, Universität Stuttgart, Stuttgart, Germany.

(13) Health Canada, Ottawa, Ontario, Canada.

(14) Läkemedelsverket, Uppsala, Sweden.

Address for correspondence

Francesco Pignatti

European Medicines Agency

7 Westferry Circus

London E14 4HB

United Kingdom

Tel.: (44 20) 75237031

E-mail: francesco.pignatti@ema.europa.eu

\section{Disclaimer}

The views expressed in this paper are those of the authors and should not be understood or quoted as the views of their respective organisations. 


\section{Introduction}

Structured frameworks for benefit-risk analysis in drug licensing decisions are being implemented across a number of regulatory agencies worldwide. The aim of these frameworks is to aid the analysis and communication of the benefit-risk assessment throughout the development, evaluation and supervision of medicines. In this review, authors from regulatory agencies, pharmaceutical companies and academia share their views on the different frameworks and discuss future directions.

\section{Background}

The balance of benefits and risks is at the heart of drug licensing decisions. According to a review of selected European agencies, licensing decisions are made intuitively, the responsibility of an accountable senior assessor or of a team, after extensive analysis of the data and discussion among experts.(1) The U. S. Food And Drug Administration (FDA) shows a similar role for clinical judgment after extensive analysis and discussion.(2) The practice has been to assess overall benefits and risks (holistically) using a prevalently intuitive approach (without precise definition of the value structure and trade-offs). As regulators in Germany described it, "First start from the benefits ('Is there a clinically significant benefit?')... if there is a clinically significant benefit..., look at adverse events. Are they acceptable for the patient?".(1)

Arguably, the robustness of the intuitive decision-making is safeguarded in a number of ways. Expertise plays a key role. Regulators typically use written reports and processes, briefings, and committee discussions until they are confident that they have determined the best course of action. External expert advice may be sought (e.g., through advisory committees) although this may sometimes be a challenge as the outside experts may be less familiar with benefit-risk judgements than the regulators. (3)

Regulators have a responsibility to communicate the benefits and risks of medicines in a way that is accessible (clear and understandable) to a broad audience to inform treatment decisions and to ensure transparency about the reasons and rationales that play a part in decisions. Regulatory agencies have published their assessment reports which include a summary of the data submitted and the conclusions. Other forms of external communication have included published perspectives or outreaching to the medical or patient community (e.g., FDA's Patient Focus Drug Development Initiative). However, accessible communication about the key benefits and risks that underlie the decisions as well as value judgments and trade-offs between benefits and risks, is often lacking. While detailed benefit-risk assessments are sometimes described in prominent publications, this is the exception much more than the rule for regulatory agencies. $(4,5)$

For instance, rimonabant (Acomplia) was authorized in the EU in 2006 for the treatment of obesity and withdrawn from the market in 2009. The benefit-risk analysis that led to the European Medicines Agency to recommend the withdrawal of the marketing authorization is described as follows: "The efficacy of rimonabant as a weight-reducing agent can be considered as moderate. The beneficial effects on cardiovascular risk factors may be of limited importance considering the reported short duration of use. (...) The expected benefits are considered as more limited compared to what was foreseen at the time of approval. (...) The absolute risk of psychiatric adverse event in clinical practice may be more common compared to what was seen at the time of approval. (...)".(6)

Eichler et al. have pointed out that regulators should consider refining their methods of assessing benefit-risk balances and switch from "implicit" to "explicit" decision making - that is, to an approach involving explicit descriptions not only of all decision criteria and interpretations of data but also valuations, such as the weighting factors for potential treatment outcomes.(7)

To address some of these methodological and communication aspects of benefit-risk assessment, drug regulators, the pharmaceutical industry and academia have acted in synergy to explore a range of available approaches (Figure 1).(8) These range from descriptive textual approaches to decisionanalytic methods that aim to quantitatively express the value judgements and trade-offs. In any approach, the aim is to facilitate the benefit-risk analysis and to better define the decision context, the drivers of the decision, and the associated uncertainty. The purpose of this paper is to describe the status of benefit-risk initiatives and plans of leading regulatory agencies as to their implementation in drug licensing decisions. We also describe two industry initiatives, and an independent approach to an overarching framework. We discuss expected changes in the near future, such as the wider implementation of structured frameworks and the need for further assessing the usefulness of quantitative decision-analysis approaches in the real-life setting. 


\section{Proposed Frameworks, Tools and Visualizations}

\subsection{The European Medicines Agency (EMA) implementation of the PrOACT- URL framework}

The EMA initiatives on benefit-risk methodology go back to 2008, when the need for a more systematic and transparent approach was recognized.(9)

A task force was set up comprising decision theorists, regulators, psychologists and statisticians, under the guidance of Lawrence Phillips, Professor of Operational Research at the London School of Economics, to identify approaches that would suit the complex organization of EMA (multiple committees with experts from several dozen organizations throughout the European Economic Area).

A stepwise approach was proposed, within a wider framework called PrOACT-URL,(10) which follows the eight-stage general decision framework proposed by Keeney et al.(11) In short, the following steps are considered:

- Problem formulation (indication, unmet need, objective of the pivotal clinical trial);

- Objectives (a full set of criteria covering the favorable and unfavorable effects, e.g., clinical efficacy and safety outcomes);

- Alternatives (treatment options being compared and regulatory options, e.g., approve or reject);

- Consequences (the magnitudes of all effects and their desirability or severity; this is summarized best in an "effects table");

- Trade-offs (the judgement about the benefit-risk balance, and the rationale for the judgement; this is best done through a multi-criteria decision analysis (MCDA) or similar method);

- Uncertainty (whatever the source);

- Risk attitude (consideration that affect attitude to risk, e.g., unmet need, may vary depending on stakeholders);

- Linked decisions (consistency with similar past decisions and impact on future decisions).

The EMA framework includes a tabular display ("effects table") of important effects and their uncertainty (Table 1). According to feedback collected from EMA's regulatory network, the table is a useful display of the key issues that can improve the transparency of the benefit-risk assessment, and support the communication among EMA's committees and the public.(12) As of 2015, the "effects table" forms an integral part of the benefit-risk section of the assessment reports of new drug applications and guidance is available for reviewers on how to use the table.(13)

While quantitative implementation of the PrOACT-URL is possible, i.e., formalizing explicitly the value structure, and the "effects table" provides the key attributes for readily initiating quantitative approaches, the version of the PrOACT-URL as applied by the EMA reviewers when assessing benefits and risks is mostly a framework that structures the assessment conceptually within the review templates, with trade-offs generally being weighed intuitively and described in qualitative terms.

There is an ongoing debate about pros and cons of decision-analysis approaches at the level of EMA's scientific committees (Table 2). Exploring real-life use of MCDA for EMA's benefit-risk assessment is being considered, particularly for complex situations (e.g., many conflicting benefits and risks, close cases, or cases where the data or clinical situation are unusual and a well-established course of action is unavailable from experience). The EMA has also been exploring MCDA to elicit value judgments from patients to inform the scientific assessment by regulators.(14)

The EMA does not impose any particular methodology to industry submitting a benefit-risk assessment as part of their drug applications. However, the EMA has encouraged applicant companies to include on a voluntary basis any decision-analysis approach, if available, to gain more experience with such approaches.

Since February 2015, the main scientific committee for human drugs (Committee for Medicinal Products for Human Use) started including the "effects table" in its assessment reports for new drug applications. The EMA is currently working on the wider implementation of the current framework across procedures and committees, including the development of further guidance for reviewers, and exploring opportunities for implementing additional tools, including methods described in the Innovative Medicines Initiative PROTECT project.(15) In terms of future directions, the EMA is 
exploring approaches to facilitate participation of patients and consumers in benefit-risk evaluation, including suitable approaches on how to capture patients' values and preferences.

\subsection{U.S. FDA Structured Approach to Benefit-Risk Assessment in Drug Regulatory Decision-Making}

Over the past several years, FDA has developed a structured approach to benefit-risk assessment in regulatory decision-making for human drug and biologic products.

The FDA Benefit-Risk Assessment Framework was developed through extensive review and analysis of previous and ongoing regulatory decisions. In 2013-2017, FDA made certain commitments to carry this work forward as part of the reauthorization of the Prescription Drug User Fee Act (PDUFA). These commitments include further development and implementation of the framework into FDA's human drug and biologic review process. Section 905 of the FDA Safety and Innovation Act of 2012 also requires FDA to implement structured benefit-risk assessment in the agency's new drug review process.

The FDA's Benefit-Risk Framework considers the following dimensions as part of the benefit-risk assessment: Analysis of Condition; Current Treatment Options; Benefit; Risk; and Risk Management. The first two dimensions, Analysis of Condition and Current Treatment Options, represent the framework's therapeutic area considerations and are distinct from the other drug-specific dimensions in the framework. The therapeutic area information represents the current state of knowledge of the condition and an assessment of the available therapies for the patient population. Both dimensions represent critical elements for establishing the context in which a regulatory decision is made, an important aspect of FDA's decisions. As knowledge and understanding of the disease improve, or as new therapies are developed, this information can be updated to establish a new context for future regulatory decisions in the therapeutic area.

The additional dimensions of Benefit, Risk, and Risk Management represent the product-specific area of the framework. The information found here relates specifically to the drug under review. As knowledge of a drug's benefits and risks changes post-approval, this information can be updated in the framework, reflecting the dynamic nature of the benefit-risk assessment during a drug's lifecycle.

Within each of these dimensions, reviewers note the relevant important evidence and uncertainties based on the current state of knowledge, relevant available information, and their review of the data. Guided questions within each dimension of the framework prompt reviewers to consider important information that could impact their assessments. Reviewers are then asked to draw conclusions within each dimension, noting important implications for the regulatory decision.

Finally, the information within each of the framework dimensions is integrated and distilled into the Benefit-Risk Summary and Assessment, a succinct well-reasoned text-based analysis that explains FDA's rationale for the regulatory decision, including important clinical judgments that contributed to the decision and the benefit-risk trade-offs that are an inherent part of regulatory decision-making. The assessment draws on the key supporting evidence and uncertainties, accounts for the understanding of the condition, and considers the available therapies that establish the context in which benefits and risks are weighed. It also includes the rationale to support the product labelling and other necessary risk management as well as post-marketing requirements/commitments if more information is necessary to further characterize the benefits or risks of the drug.

During the agency's development of the framework, FDA recognized that a critical element of widely adopting a structured approach to benefit-risk assessment was integration into existing work processes and products. In March 2015, FDA began using a newly revised template for clinical reviews that incorporates the Benefit-Risk Framework as part of the executive summary of the review. This template is currently being used for new molecular entity new drug applications (NME NDAs) and original biologics license applications (BLAs), as part of a staged implementation plan. An incremental implementation plan allows opportunity for continued refinement of the framework and its integration into the human drug review process before further expansion into additional types of applications. As the benefit-risk assessment is revisited in the post-market setting based on new information for these applications, review teams analyzing the safety issue will be expected to update the benefit-risk framework with the analysis conducted, including any regulatory action resulting from that work, as appropriate. 


\subsection{The Japanese PMDA Points to Be Considered by the Review Staff Involved in the Evaluation Process of New Drug}

Currently, the benefit-risk "framework" for the assessment of marketing authorization applications for new drugs follows the "Points to Be Considered by the Review Staff Involved in the Evaluation Process of New Drug" in 2008.(16) This document uses a check-list approach and summarizes the points that need to be considered during the actual assessment process of new medical products (Figure 2). The points concerning benefit-risk include the following:

- Has the efficacy been clearly confirmed?

- Have factors related to the recognized risk been clearly identified?

- Has any effective treatment been identified to prevent/inhibit occurrence of the recognized risk?

- Is the recognized risk acceptable, even if it is serious, when considering the benefits?

The regulatory decisions on each consideration points are described in the review report.

In the post-authorization stage a re-examination system plays an important role. A new medicinal product containing a new active ingredient is re-examined 8 years after approval as new product. All the collected post-marketing data are assessed on the basis of benefit-risk balance to reconfirm the clinical effectiveness of the drug. The assessment report of re-examination is published. Furthermore, for important issues regarding efficacy or safety and regulatory actions taken in the post-approval stage, PMDA publishes the assessment report even earlier than 8 years post-approval.

The research group on the Japanese Risk Management Plan (J-RMP) was established in April 2012 supported by the Ministry of Health, Labour and Welfare research fund to explore implementation of effective RMP in Japan. Researchers from academia, industry and regulators from PMDA participate in this group and the study projects include how to assess benefit-risk in line with J-RMP. J-RMP guidance came into force in April 2013.(17) In J-RMP, the benefit-risk assessment is required at selected milestones. RMPs are continuously updated and additional actions are taken as necessary. The research group is now discussing the methods of benefit-risk assessment. In 2015, future directions about what should be done in Japan for the improvement of benefit-risk assessment will be suggested by the research group.

\subsection{International regulators initiatives and the Centre for I nnovation in Regulatory Science ( CI RS) Universal Methodology for Benefit Risk Assessment (UMBRA)}

Since its inception in 2001, the CIRS has been actively involved in investigating ways that companies, regulatory agencies, and other stakeholders approach the analysis and consequent decisions about the benefits and risks of medicines.

Regulatory initiatives in Australia, Canada, Singapore and Switzerland: The Consortium on Benefit-Risk Assessment (COBRA) and further collaborations

The Consortium on Benefit-Risk Assessment represents a group of like-minded regulators representing Australia, Canada, Singapore and Switzerland, who believe that they can each improve their efficiency by leveraging efforts towards similar goals. Since 2008, a series of projects was started, aiming to allow these regulators to exchange review reports and use these reports in their own. One of those projects was the development of a benefit risk assessment template ("COBRA Benefit Risk Template"). CIRS worked closely, over a period of 5 years, with these agencies to conceptualize, refine and pilot a standardized descriptive methodology approach. $(8,18)$ The approach was originally based on the EMA guidance document of 2008 (19) and has evolved since to comply with the UMBRA framework (see below).(20)

Following the experience with the COBRA Benefit Risk Template, members of the consortium have continued to collaborate on inclusion of a benefit risk section in their assessment templates. In general, members currently favor a qualitative approach to the description of benefits and risks, and do not include any specific visualization techniques in their templates. As benefit-risk assessment evolves, this may change. The current project on benefit risk envisages the agencies having a common section on benefit risk assessment in their respective assessment report templates within 2015. Following this, the agencies intend to evaluate possibilities for a common public assessment report template. 
The initial incentive for development of a common benefit risk assessment template was to facilitate sharing these assessment reports among the agencies. The ability of regulators to share assessment reports depends on factors including their respective legal frameworks, availability of a suitable electronic system, and the ability to use these reports as part of their decision frameworks. Issues related to efficient sharing of assessment reports are being addressed in a variety of multinational fora including the International Consortium of Medicines Regulatory Agencies (ICMRA), the International Generic Drug Regulators Pilot (IGDRP), among others.

\section{Regulatory initiatives in Chinese Taipei, China, Indonesia, Malaysia, Singapore and Philippines}

A number of regulatory agencies are working with CIRS to pilot a summary version of the COBRA Benefit Risk Template as one aspect of their quality decision making processes. This CIRS initiative (international Summary Approach to Benefit Risk Evaluation, iSABRE), is based on the recognition that integrating a standardized approach to benefit-risk assessment into the regulatory review process is not only a facilitator of good review practices, but encourages an overarching approach to quality decision making. CIRS has completed an initial pilot and this has been evaluated by Chinese Taipei, China, Indonesia, Malaysia, Singapore and Philippines and is under consideration for evaluation by Saudi Arabia (SFDA) and South Africa. These agencies are characterized by their use of different review models and these pilot studies will help to determine the generalizability of the iSABRE approach in a broader context of drug development and assessment.

\section{The CIRS UMBRA}

The UMBRA was developed by CIRS based on the realization that a high degree of consistency has been observed across the frameworks developed by regulators and industry, with each sharing eight common elements: a decision context; the ability to build a value tree; refining the value tree; assessing the relative importance of each benefit and risk; evaluating and scoring against other treatment options; evaluating uncertainty around the information; the concise presentation of resultswith the option of using visualizations; and ultimately, applying expert judgment to make and communicate the outcome.(20)

The common elements of UMBRA can be applied to the development of any number of assessment tools, each providing a consistent and transparent approach to the appraisal of medicines during development, regulatory review and post-approval surveillance. For example, the EMA, FDA, and the Benefit-risk Action Team (BRAT) frameworks can each be mapped to the specific elements of the UMBRA framework. CIRS has been advocating the BRAT process in constructing the benefit-risk section of the Common Technical Document used by companies when submitting drug applications to regulatory authorities, with UMBRA serving as the common framework upon which the benefit-risk section of the submission can be built. CIRS advocates that all new tools and processes developed to assess and communicate the benefits and risk of medicines should map to UMBRA to ensure a common regulatory language for the systematic, structured approach to benefit risk assessment.

\subsection{The Pharmaceutical Research Manufacturers of America (PhRMA) BRAT Framework}

The BRAT Framework was co-developed by PhRMA and several PhRMA member companies and traces its origins to 2005, when PhRMA proposed a project to review regulatory approaches to benefit-risk assessment, with an eye to recommending a transparent, systematic approach useful to structuring and facilitating benefit-risk decision-making. Informed by principles of decision science, the BRAT Framework is guided by a number of precepts: a systematic approach to defining the decision context and outcomes, documentation of underlying assumptions, transparency of summarization measures, the flexibility to accommodate differing technical benefit-risk methodologies and perspectives, and the use of visual displays to communicate complex data. The resulting BRAT Framework is best characterized as a general platform for benefit-risk assessment that facilitates the selection, organization, summarization, and interpretation of evidence relevant to benefit-risk decisions.

Since its inception, the BRAT Framework has been iteratively developed and tested, first by the BRAT in conjunction with member PhRMA companies, in which the framework was tested using hypothetical drugs and simulated scenarios that encompassed the challenges and realities typically encountered in pharmaceutical development and benefit-risk assessment. $(21,22)$ In this setting, the framework was 
effective in facilitating benefit-risk decision making. Subsequently, a voluntary pilot program was conducted among PhRMA members to evaluate the framework's real-world utility, flexibility, and value. Upon conclusion of the pilot program, in which multiple companies applied the BRAT Framework to products of their choice, pilot program participants unanimously found the framework to be effective and flexible, with applicability to a broad range of circumstances.(23) Beyond the pilot, the framework has also been used independently by BRAT members and others in various applications and public meetings, including FDA Advisory Committee meetings and periodic benefit-risk evaluation reports. (24-27)

The BRAT framework formally consists of the six steps in Table 3, though it has been modified and extended by individual companies that have implemented custom versions of BRAT. These steps and the BRAT documentation are highly focused on medical treatments, though they are based on principles found in general decision analysis approaches. The framework applies to both development and post-approval assessments and can be used by both sponsors and health authorities.

Particular novel highlights of the BRAT Framework include

- Documenting the rationale for all key components of the decision, such as the decision to include or exclude particular endpoints. This documentation is particularly valuable for the current ICH and EMA requirements for Periodic Benefit-risk Evaluation Reports (PBRERs), which require explicitly specifying those benefits and risks considered key to the assessment.

- Allowing for multiple versions of a value tree of key endpoints needed for the assessment depending on data availability, the identified vs. potential nature of the endpoints, and the level of detail needed for the audience.

- Customizing the framework, to account for the differences between what would ideally be used in a benefit-risk assessment and what information is measurable and available. This step is particularly critical in post-approval assessment, where data are collected from multiple sources with different endpoints and varying levels of quality.

- Tabular and graphic displays that clearly depict difference between treatments in all benefits and harms included in the value tree. Key benefit-risk summary tables summarize the treatment effects and potential risk differences or other measures for all benefits and harms in one table (Figure 3). These tables are similar to the "effects table" used in PrOACT-URL. Risk difference forest plots summarize large amounts of information on treatment differences for multiple benefits and harms (Figure 4). These displays support rapid interpretation of information on multiple outcomes. (21)

- While no particular means of weighting endpoints is proscribed in the BRAT framework, the value tree and key benefit-risk summary table provide the requirements for readily initiating quantitative approaches.

The software released with the BRAT Framework allows for creation of value trees, key benefit-risk summary tables with graphic annotations, and risk difference or relative risk forest plots. The current tool supports dichotomous endpoints only.

Several companies have implemented their own version of BRAT, borrowing ideas from the original BRAT work and extending it with their existing processes or requirements. The BRAT software is in use, though several companies have developed their own versions with additional capabilities.

In 2012, the BRAT Framework was transitioned to the CIRS (see section 3.4). The BRAT documentation and software are available at the CIRS site (www.cirs-brat.org).

\subsection{The World Self-Medication Industry tools for the Benefit-Risk Assessment of Nonprescription Drugs (BRAND tools)}

The availability of nonprescription drugs is an important component of efficient healthcare delivery as it facilitates appropriate self-management by consumers and thus improves the utilization of traditional healthcare resources. The principles of benefit-risk assessment for nonprescription drugs are the same as for prescription drugs. However, their proper application requires explicit incorporation of the unique context in which nonprescription drug decisions are made. For example, the regulatory consideration will often be whether a specific drug should have nonprescription vs prescription marketing authorization. Further, this evaluation will often be conducted after the drug has had prescription marketing authorization for some period of time. In this case the benefit-risk assessment is based on the incremental benefits and incremental risks that differentiate prescription and nonprescription use of the drug. This incremental benefit-risk will be driven by how consumers behave 
when deciding to use the drug and over the course of therapy (Table 4). These behaviors are influenced by labelling of the nonprescription product and perhaps pharmacists input. Specifically, while use of nonprescription drugs under a collaborative care model between consumer and pharmacist is encouraged, the drugs are often used without the assessment and instructions of a healthcare professional prescriber.

In consideration of the above, the World Self-Medication Industry contracted with outside experts (Eric P. Brass, Ragnar Lofstedt and Ortwin Renn) to develop benefit-risk tools optimized for regulatory decision making involving nonprescription drugs. The resulting Benefit-Risk Assessment for Nonprescription Drugs (BRAND) tools have been published $(28,29)$ and seen application in a variety of settings. The tools begin with a value-tree(21, 22, 30) modified to include common benefit-risk domains for nonprescription drugs in order to comprehensively identify all benefit and risk attributes which have the potential to contribute to the drug's net benefit-risk.(28) This value-tree is designed to be completed early in development and facilitate alignment amongst all stakeholders, including regulators. An evaluation matrix is then applied to each attribute which independently assessed the frequency at which the attribute is likely to occur in the nonprescription setting and the clinical consequences of the event should it occur.(29) In this way commonly occurring attributes that have no clinical sequelae can be differentiated from attributes likely to contribute to incremental benefit or risk. This evaluation matrix should be completed early in development to identify critical data gaps that contribute uncertainty to the assessment and must be addressed during the development program. At the conclusion of the development program the evaluation matrix is again completed to provide a final overall benefit-risk profile.(29) To ensure the longitudinal application of the tools, and to maximize communication and transparency, it is recommended that an integrative approach such as the International Risk Governance Council framework(31) be utilized.(28) The BRAND tools are intended to be flexible and non-proscriptive to accommodate varied cultures and statutes in different regulatory jurisdictions.

Since their introduction the principles underlying the BRAND tools have seen wide acceptance and been applied implicitly or explicitly in sponsor-regulator discussions, communications and submissions. For example, both the United Kingdom's Medicines and Healthcare products Regulatory Agency and the New Zealand Medicines and Medical Devices Safety Authority have incorporated aspects of the BRAND tools into their guidance on classification of medicines. $(32,33)$ Importantly, sponsors have used the tools in internal exercises to better understand the needs of nonprescription development programs prior to initiating programs and to aid in early communications with regulators.

Of note, while the above discussion focuses on the evaluation of switching a drug from prescription to nonprescription status, the BRAND tools apply equally well to the post-approval assessment of an existing nonprescription drug. Indeed, the components of the BRAND tools may also be useful in the assessment of some prescription drugs or medical devices as the underlying principles of longitudinal application, emphasis on communication and transparency, comprehensive attribute identification, and evaluation that reflects both frequency and clinical impact are highly generalizable.

The future utility of the BRAND tools will likely be dependent on sponsors investing the time in rigorous application of the value-tree and scoring matrix tools to characterize drugs under evaluation. Use of the tools to present the benefit-risk assessment will force data-driven annotation of entries, increasing acceptance by regulators and other stakeholders. Further, experience will make clear that the scoring matrix is not an absolute quantitative plus/minus summary, but rather a tool for providing an overall profile and highlighting the major drivers of benefit-risk. For example in a recent exercise, application of the BRAND tools to nonprescription diclofenac identified an increased rate of cardiovascular events as a major potential risk and guided the development of a potential risk mitigation strategy to reduce the frequency score in the scoring matrix.(34)

\section{Discussion}

The structured frameworks that are being implemented at various regulatory agencies reflect common objectives, namely to guide the analysis and communication of benefits, risks and trade-offs for medicin 
al products. If adhered to, the frameworks can help setting internal standards and consistency for decision-making, enhancing the clarity of the decision-making process, encouraging the use of appropriate documentation and a systematic articulation of each benefit and risk and their relative importance, providing consistency of communication and visualization of benefits and risks to various stakeholders. This is expected to increase transparency and to facilitate the benefit-risk assessment process. Structured frameworks might also help increasing alignment between sponsors and regulators around critical data elements needed.

Tables and figures can be used when communicating benefits and risks to a variety of audiences with a minimum statistical and medical background. Additional visualizations that may enhance communication of benefits and risks for some audiences include network graphs, forest plots, interval plots.(35) The frameworks promote a deconstructed approach and encourage analytic thinking, or visual approaches that may facilitate intuitive thinking, or a combination of the two as in the CIRSBRAT framework.

With practice, more detailed, possibly even disease-specific, frameworks could be explored. Similar to defining objectives and endpoints in clinical trials, more specific frameworks describing the criteria that are important to assess benefits and risks in a particular setting could be developed. The tools developed for nonprescription medicines described above are an example of a more specific decision framework that has been gaining regulatory acceptance. $(28,32)$

Currently, no single framework has been agreed among all regulators. However, in view of the many similarities, further harmonization is possible, as reported by CIRS, the Innovative Medicines Initiative PROTECT project 2012 and others.(8, 35-37) Harmonization may also affect the way applicant companies structure their benefit-risk assessment in regulatory submissions. To this end, an International Conference on Harmonisation of Technical Requirements for Registration of Pharmaceuticals for Human Use Expert Working Group is currently evaluating and revising its guidance and documentation for benefit-risk assessment within the Common Technical Document, M4E(R1) (Section 2.5.6). The revision is expected to include greater specificity on the format and structure of benefit-risk information with the goal of harmonizing the presentation of this information in regulatory submissions. Revised guidance is expected by the end of 2016. Again, although currently no single framework or decision-analysis method is likely to be accepted by all regulators, it is likely that with further experience, additional guidance and best practices will become available.

One of the challenges for any framework aiming to describe the benefit risk assessment is the expression of value trade-offs. EMA recommends reviewers to describe the importance of different effects (a qualitative description of how much the reviewers value the effects, expressing "value judgements") and to compare the effects among each other. The guidance recommends describing what the minimally clinically relevant effect worth detecting is, and then comparing the observed results against that threshold. For example, "Generally, given the poor prognosis in terms of survival in the context of this advanced cancer setting, an improvement in median overall survival in the order of 2-3 months is considered of clinical relevance. The 6 month difference in median overall survival observed was considered to be very important from a clinical point of view". A similar approach can be used to describe risks and trade-offs, defining what are acceptable and tolerable risks. (38) Similarly, FDA recommends that reviewers clearly communicate the relative importance of the issues and how they were weighed in the decision. The approaches described in the BRAND tools use a semiquantitative rating of the importance to illustrate the contribution of each attribute.(29) Once the different effects are ranked according to their importance, value trade-offs can be described along the hierarchy of effects by stating what would be the maximum loss that one is willing to accept in order to improve likelihood of achieving a gain on the next most important effect. Further, this may guide risk mitigation strategies and prioritize any post-marketing assessments. However, even using these approaches, analysis and communication of the value structure and trade-offs remains inherently difficult in complex situations, e.g. there are multiple conflicting attributes with no clearly outstanding effects, where the benefit-risk assessment requires deep reflection.

Quantitative decision-analysis approaches such as MCDA that incorporate explicit preference information and trade-offs have been explored within structured frameworks as aids to decisionmaking as well as communication.(35, 39-44) A number of case studies using MCDA implementations, including stochastic multi-criteria acceptability analysis (SMAA) and value theory for the benefit-risk assessment of rimonabant, telithromycin, efalizumab, natalizumab and warfarin have been recently published. $(15,40)$ Preferences studies can yield numeric weights that can also augment any of the descriptive frameworks. Currently, however, regulators are still debating the usefulness of such methods in making or communicating values and decisions in the regulatory context. More experience is needed in order to determine the real value of quantitative decision-analytic methods in the regulatory setting. 
Finally, an outstanding question today is to what extent structured frameworks have a positive real world impact on benefit-risk decision-making and communication. Although a number of favorable impacts are expected in terms of aiding the assessment and its communication, and the initial feedback from regulators that have implemented structured frameworks is encouraging, it is probably too early for any firm conclusions, as the implementation of these approaches in the regulatory setting is still ongoing. For all the frameworks proposed, it is clear that the different stakeholders need more experience. Sponsors must invest additional time to explore different approaches in the context of regulatory submissions as no single framework or method is likely to be accepted in its current form by all regulators. Likewise, regulators acknowledge that using a structured approach requires additional time to complete, but there is recognition that a structured approach is generally a better way to communicate a regulatory decision. As regulators continue with implementation, they will learn what features work best in their jurisdictions and within their established procedures in an iterative process. Lastly, all stakeholders should continue to engage with each other and explore opportunities for further optimization and harmonization of structured approaches to benefit-risk assessment. 


\section{References}

1. European Medicines Agency. Benefit-risk methodology project: Work package 1 report: description of the current practice of benefit-risk assessment for centralised procedure products in the EU regulatory network. 2011.

2. FDA. Structured Approach to Benefit-Risk Assessment in Drug Regulatory Decision-Making. FDA; 2013 [updated February, 2013]; Available from: http://www.fda.gov/downloads/Forl ndustry/UserFees/PrescriptionDrugUserFee/UCM329758. pdf.

3. Brass EP. Assessing the benefit-risk for new drugs: are the FDA's Endocrinologic and Metabolic Drugs Advisory Committee and the Division of Metabolism and Endocrinology Products in sync? Diabetes care. 2013;36(7):1823-6. Epub 2013/05/23.

4. Unger EF. Weighing benefits and risks--the FDA's review of prasugrel. The New England journal of medicine. 2009;361(10):942-5. Epub 2009/09/04.

5. Beasley BN, Unger EF, Temple R. Anticoagulant options-- why the FDA approved a higher but not a lower dose of dabigatran. The New England journal of medicine. 2011;364(19):1788-90. Epub $2011 / 04 / 15$.

6. $\quad$ European Medicines Agency. Assessment report for Acomplia. 2009; Available from: http://www.ema.europa.eu/docs/en_GB/document_library/EPAR_-_Assessment_Report__Variation/human/000666/WC500021280.pdf.

7. Eichler HG, Abadie E, Baker M, Rasi G. Fifty years after thalidomide; what role for drug regulators? British journal of clinical pharmacology. 2012; 74(5):731-3. Epub 2012/03/01.

8. Liberti L, McAuslane N, Patel P, Breckenridge A, Eichler HG, Peterson R. Regulatory review: How do agencies ensure the quality of decision making? Clinical pharmacology and therapeutics. 2013; 94(3):305-8. Epub 2013/08/22.

9. Eichler HG, Abadie E, Raine JM, Salmonson T. Safe drugs and the cost of good intentions. The New England journal of medicine. 2009;360(14):1378-80. Epub 2009/04/03.

10. Phillips LD, Fasolo B, Zafiropoulous N, Beyer A. Is quantitative benefit-risk modelling of drugs desirable or possible? Drug Discovery Today: Technologies. 2011;8(1).

11. Hammond J, Keeney R, Raiffa H. Smart choices: A practical guide to making better decisions. Boston: Harvard University Press; 1999.

12. European medicines Agency. Benefit-risk methodology project. Update on work package 5: Effects Table pilot (Phase I). 2014.

13. European Medicines Agency. Assessment templates and guidance. 2015.

14. Agency EM. Regulatory and methodological standards to improve benefit-risk evaluation of medicines2014. Available

from: http://www.ema.europa.eu/docs/en_GB/document_library/Report/2014/04/WC500165803. pdf.

15. Innovative Medicines I nitiative PROTECT Project. 2012 [10/22/2012]; Available from: http://www.imi-protect.eu/index.shtml.

16. Pharmaceuticals and Medical Decvices Agency. Points to Be Considered by the Review Staff Involved in the Evaluation Process of New Drug2008. Available from: http://www.pmda.go.jp/english/service/good review practice.html. 
17. Pharmaceuticals and Medical Decvices Agency. Risk Management Plan Guidance2012. Available from: http://www.pmda.go.jp/english/service/rmp.html.

18. Walker S. Implementing an internationally acceptable framework for the benefit-risk assessment of medicines: How close are we to this objective? CIRS Workshop J une 20132014.

19. European Medicines Agency. Reflection paper on benefit-risk assessment methods in the context of the evaluation of market authorisation applications of medicinal products for human use2008. Available from:

http//www.ema.europa.eu/docs/en GB/document library/Regulatory and procedural guideline/2010/ 01/wc500069634.pdf.

20. Dombrowski C. International Group Finds Harmony In Benefit-Risk Assessment Framework. The Pink Sheet. 2012 August 27.

21. Levitan BS, Andrews EB, Gilsenan A, Ferguson J, Noel RA, Coplan PM, et al. Application of the BRAT framework to case studies: observations and insights. Clinical pharmacology and therapeutics. 2011;89(2):217-24. Epub 2010/12/24.

22. Coplan PM, Noel RA, Levitan BS, Ferguson J, Mussen F. Development of a framework for enhancing the transparency, reproducibility and communication of the benefit-risk balance of medicines. Clinical pharmacology and therapeutics. 2011;89(2):312-5. Epub 2010/12/17.

23. Noel R, Herman R, Levitan B, Watson DJ, Van Goor K. Application of the Benefit-Risk Action Team (BRAT) Framework in Pharmaceutical R\&D: Results From a Pilot Program. Drug Information Journal. 2012;46(6): 736-43.

24. Brown DM, Nguyen QD, Marcus DM, Boyer DS, Patel S, Feiner L, et al. Long-term outcomes of ranibizumab therapy for diabetic macular edema: the 36-month results from two phase III trials: RISE and RIDE. Ophthalmology. 2013;120(10):2013-22.

25. Rivaroxaban Cardiovascular and Renal Drugs Advisory Committee Briefing Document September 8, 2011. 2011; Available from: http:// www.fda.gov/downloads/AdvisoryCommittees/CommitteesMeetingMaterials/Drugs/Cardio vascularandRenalDrugsAdvisoryCommittee/UCM272005.pdf.

26. Belatacept Cardiovascular and Renal Drugs Advisory Committee Briefing Document March 1, 2010 2010; Available from: http://www.fda.gov/downloads/AdvisoryCommittees/CommitteesMeetingMaterials/Drugs/Cardio vascularandRenalDrugsAdvisoryCommittee/UCM203517.pdf.

27. Rivaroxaban Cardiovascular and Renal Drugs Advisory Committee Briefing Document March 19, 2009 2009; Available from: http://www.fda.gov/downloads/AdvisoryCommittees/CommitteesMeetingMaterials/Drugs/Cardio vascularandRenalDrugsAdvisoryCommittee/UCM143666.pdf.

28. Brass EP, Lofstedt R, Renn O. Improving the decision-making process for nonprescription drugs: a framework for benefit-risk assessment. Clinical pharmacology and therapeutics. 2011;90(6):791-803. Epub 2011/11/04.

29. Brass EP, Lofstedt R, Renn O. A decision-analysis tool for benefit-risk assessment of nonprescription drugs. Journal of clinical pharmacology. 2013;53(5):475-82. Epub 2013/02/06.

30. Von Winterfeldt D EW. Decision analysis and behavioural research. Cambridge Cambridge University Press; 1986. 
31. Renn O. IRGC, International Risk Governance Council. Risk Governance: Towards an integrative approach. White Paper No. 1. Geneva2005.

32. Medicines and Healthcare Products Regulatory Agency. How to change the legal classification of a medicine in the UK.

33. New Zealand Medicines and Medical Devices Safety Authority. How to change the legal classification of a medicine in New Zealand. Available from: http://www.medsafe.govt.nz/downloads/How_to_change_medicine_classification.pdf.

34. Brass EP, Swanson J, Weisman S. Benefit-risk assessment of nonprescription diclfoenac. International Conference on Pharmacoepidemiology \& Therapeutic Risk Management [in press]2015.

35. Mt-Isa S, Wang N, Hallgreen CE, Callréus T, Genov G, Hirsch I, et al. Review of methodologies for benefit and risk assessment of medication. 2013.

36. Walker S, McAuslane N, Liberti L, Leong J, Salek S. A universal framework for the benefit-risk assessment of medicines : Is this the way forward?. Therapeutic Innovation and Regulatory Science. 2014(September).

37. Levitan B, L P, Walker S. Structured Approaches to Benefit-Risk Assessment: A Case Study and the Patient Perspective. Therapeutic Innovation \& Regulatory Science. 2014;48: 564-73.

38. European Medicines Agency. Guidance document on the content of the <Co-> Rapporteur day 80 critical assessment report. 2014; Available

from: http://www.ema.europa.eu/docs/en_GB/document_library/Regulatory_and_procedural_guidelin e/2009/10/WC500004800.pdf.

39. Guo JJ, Pandey S, Doyle J, Bian B, Lis Y, Raisch DW. A review of quantitative risk-benefit methodologies for assessing drug safety and efficacy-report of the ISPOR risk-benefit management working group. Value Health. 2010; 13(5):657-66. Epub 2010/04/24.

40. Hughes D, Waddingham EAJ, Mt-Isa S, Goginsky A, Chan E, Downey G, et al. I MI-PROTECT Benefit-Risk Group. RECOMMENDATIONS REPORT. Recommendations for the methodology and visualisation techniques to be used in the assessment of benefit and risk of medicines. 2013.

41. Sashegyi A, Felli J, Noel R. Benefit-Risk Assessment in Pahrmaceutical research and Development2014.

42. Phillips LD. Benefit-risk modeling of medicinal products: Methods and applications. In: Sashegyi A, Felli J, Noel R, editors. Benefit-Risk Assessment in Pahrmaceutical research and Development. Boca Raton: CRC Press; 2014.

43. European Medicines Agency. Benefit-risk methodology project: Work package 2 report: Applicability of current tools and processes for regulatory benefit-risk assessment 2010.

44. Gingery D. Systematizing Patient Views Unlikely, FDA's Temple Says. The Pink Sheet. 2014 February 24. 


\section{Abbreviations:}

PhRMA BRAT: Pharmaceutical Research and Manufacturers of America Benefit-Risk Action Team;

CIRS, Centre for Innovation in Regulatory Science;

UMBRA, Unified Methodology for Benefit-Risk Assessment;

CASS Taskforce of representatives from Health Canada, Australia's Therapeutic Goods Administration, Swissmedic and the Singapore Health Science Authority;

COBRA, Consortium on Benefit-Risk Assessment;

SABRE, Southeast Asia Benefit Risk Evaluation;

EMA, European Medicines Agency;

US FDA, United States Food and Drug Administration;

WSMI BRAND, World Self-Medication Industry Benefit-Risk Assessment for Nonprescription Drugs;

IMI PROTECT WP5, Innovative Medicine Initiative "Pharmacoepidemiological Research on Outcomes of Therapeutics by a European ConsorTium", work package 5;

Advance, "Accelerated development of vaccine benefit-risk collaboration in Europe". 
Figures and tables

Figure 1: Summary of main benefit-risk initiatives (Modified from M. Ouwens et al., ESFPI / PSI Benefit-Risk Special I nterest Group meeting 2013.)

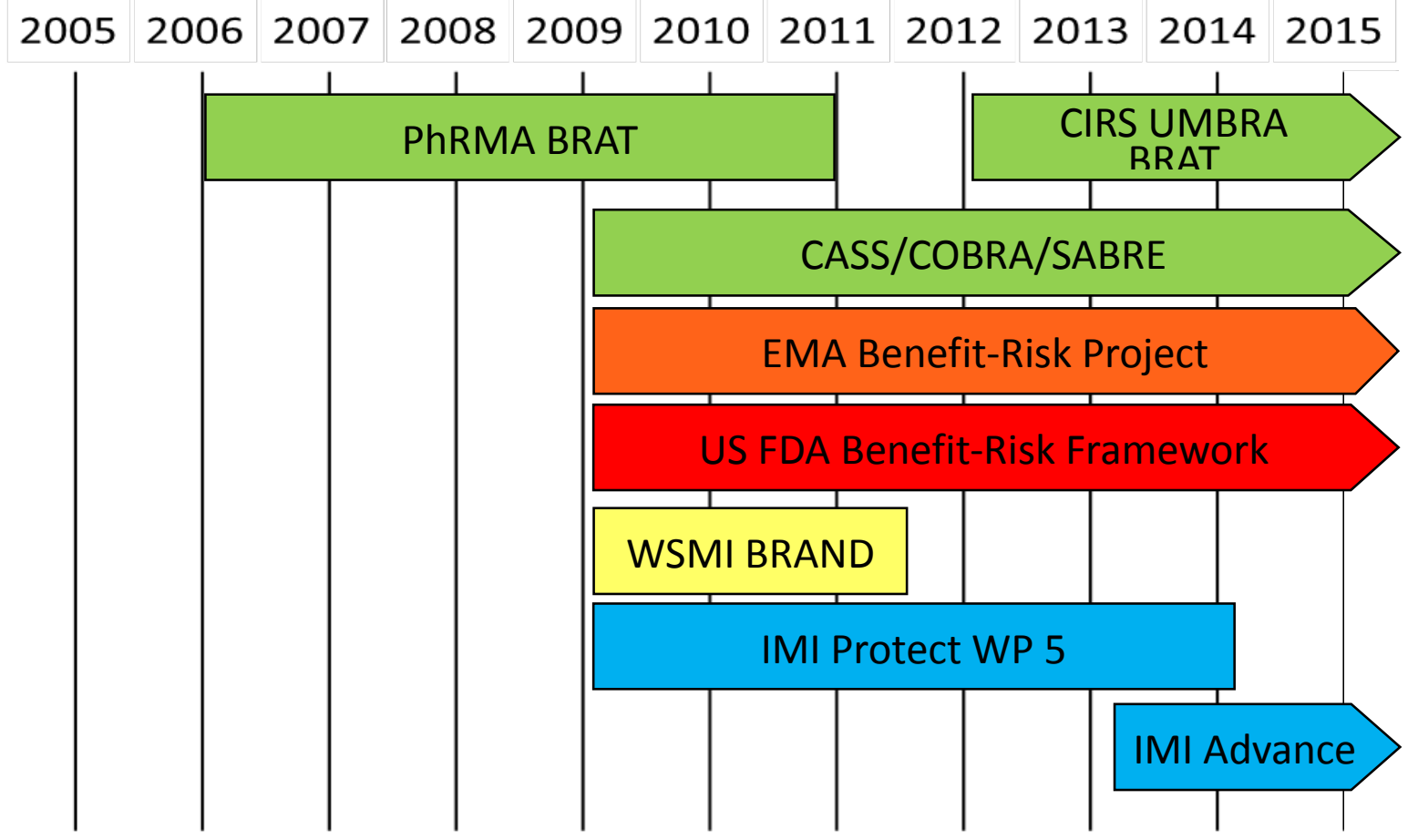




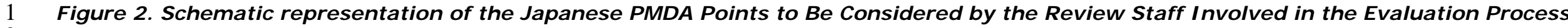
of New Drug.(16)

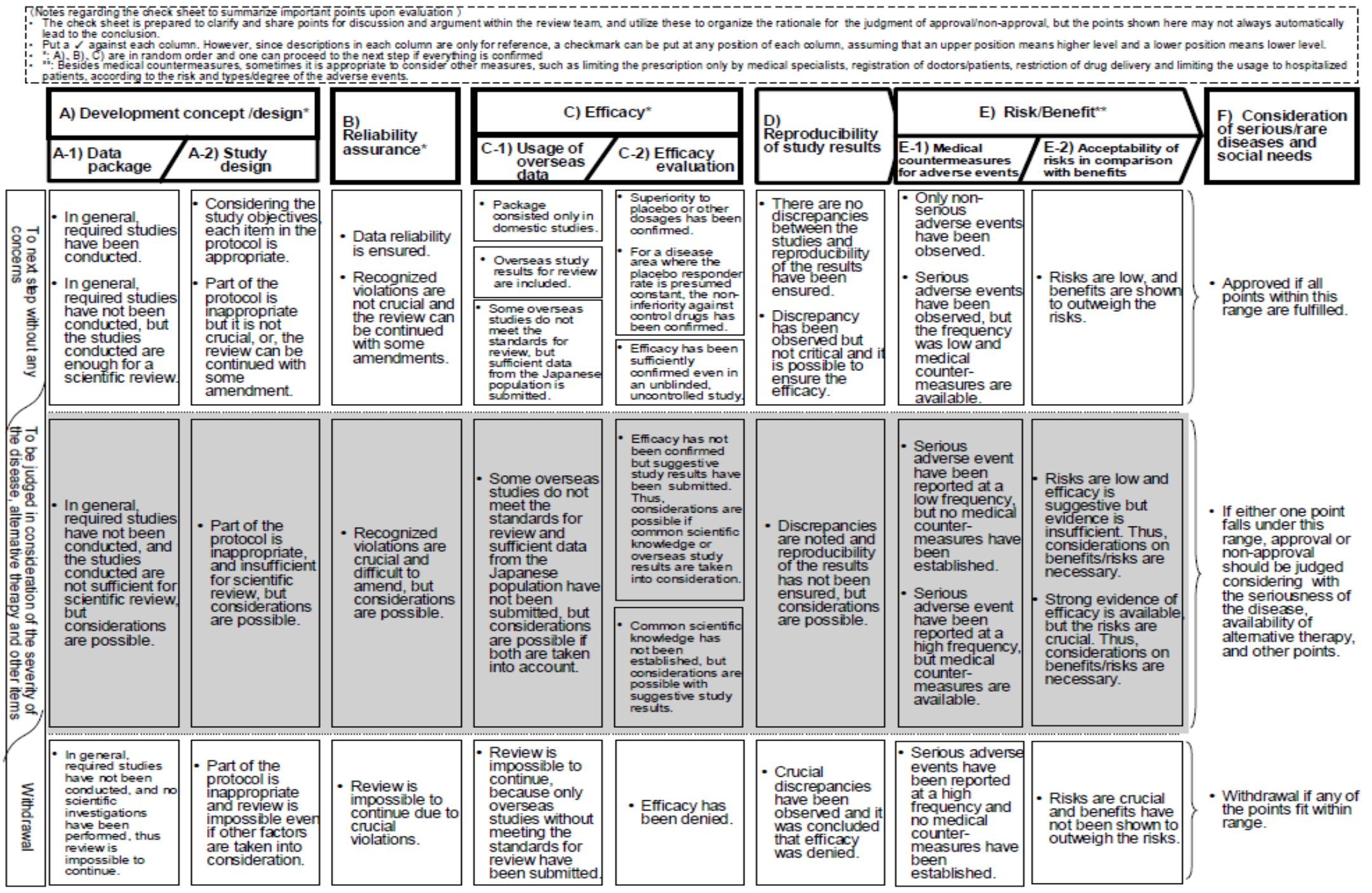


Figure 3: Key benefit-risk summary table for statins for congestive heart failure example of the BRAT framework.

\begin{tabular}{|c|c|c|c|c|c|c|c|}
\hline \multirow{7}{*}{ 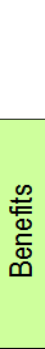 } & & Outcome & $\begin{array}{l}\text { Study } \\
\text { Drug } \\
\text { Risk }^{\mathrm{a}}\end{array}$ & \multirow{2}{*}{$\begin{array}{c}\text { Placebo } \\
\text { Risk }^{\mathrm{a}} \\
64\end{array}$} & \multicolumn{2}{|c|}{$\begin{array}{c}\text { Risk Difference } \\
\text { per } 10,000 \text { person-yrs }\end{array}$} & \multirow[t]{2}{*}{ Risk Difference Forest Plot } \\
\hline & \multirow{4}{*}{$\begin{array}{c}\text { Cardio- } \\
\text { vascular Issues }\end{array}$} & Angina requiring $C A B G$ & 3.7 & & -2.6 & $(-6.4,1.2)$ & \\
\hline & & Coronary heart dise ase de ath & 31.0 & 33.6 & -2.7 & $(-16.9,11.6)$ & \\
\hline & & Lipid levels meet target & 6700 & 2900 & 3800 & $(2,691,4,909)$ & \\
\hline & & Nonfatal myocardial infarction & 22.1 & 43.3 & -21.2 & $(-95.2,52.8)$ & $\Delta$ \\
\hline & & Fatal ischemic stroke & 18.6 & 35.4 & -16.8 & $(-29.9,-3.6)$ & $\Delta$ \\
\hline & Ischem Ic Stroke & Nonfatal ischemic stroke & 97.5 & 119.8 & -22.3 & $(-39.8,-4.8)$ & $\square$ \\
\hline & & & & & & & \\
\hline & liver Damage & Liver failure & 0.6 & 0.6 & 0.0 & $(-1.6,1.6)$ & \\
\hline & LIver Lamage & Persistently elevated transaminases & 13.6 & 10.1 & 3.5 & $(-3.8,10.9)$ & 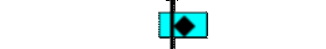 \\
\hline 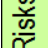 & & Myopathy & 5.9 & 5.3 & 0.6 & $(-4.5,5.6)$ & \\
\hline & Damage & Rhabdomyolysis & 0.6 & 0.5 & 0.1 & $(-1.5,1.6)$ & \\
\hline & & Severe mabdomyolysis ? kidney failure & 0.029 & 0.026 & 0.003 & $(-0.07,0.08)$ & \\
\hline
\end{tabular}

Figure 4: Risk difference forest plot for triptans for migraine example.(21)

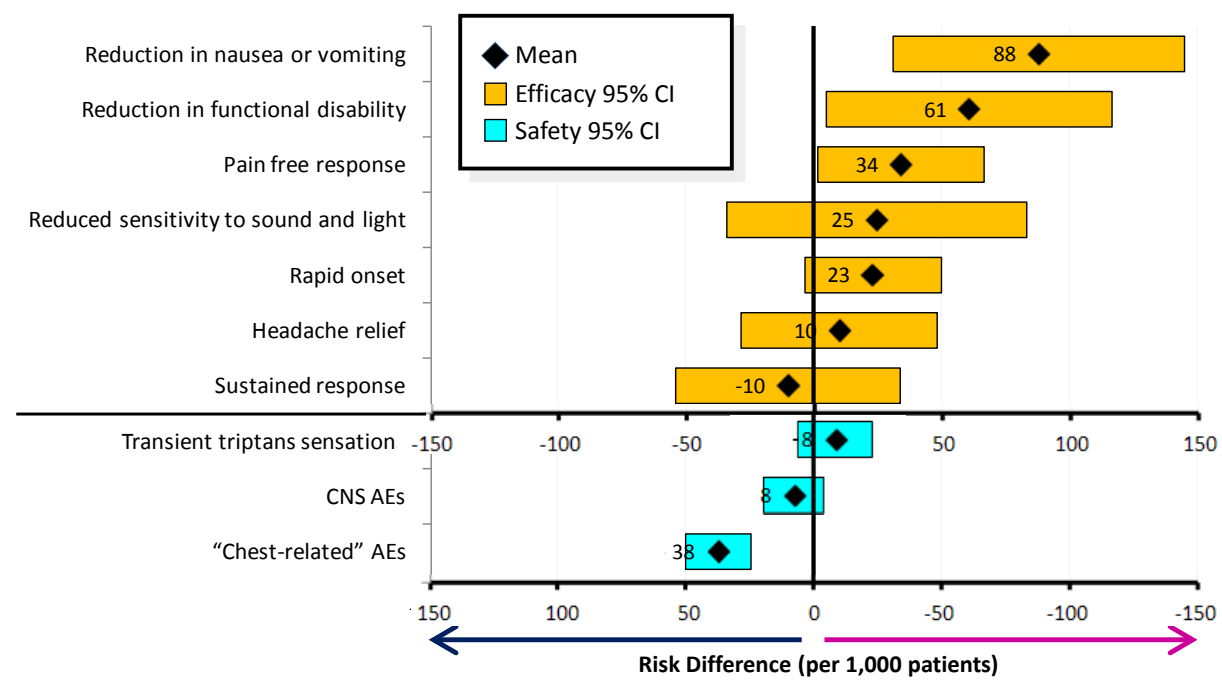

Favors comparator Favors study drug 
Table 1. Hypothetical example of an "effects table" for lixisenatide for treatment of type 2 diabetes mellitus (the data in this example are based on the European Public assessment report $\mathrm{EMEA} / \mathrm{H} / \mathrm{C} / 002445$ available on the EMA website www.ema.europa.eu)

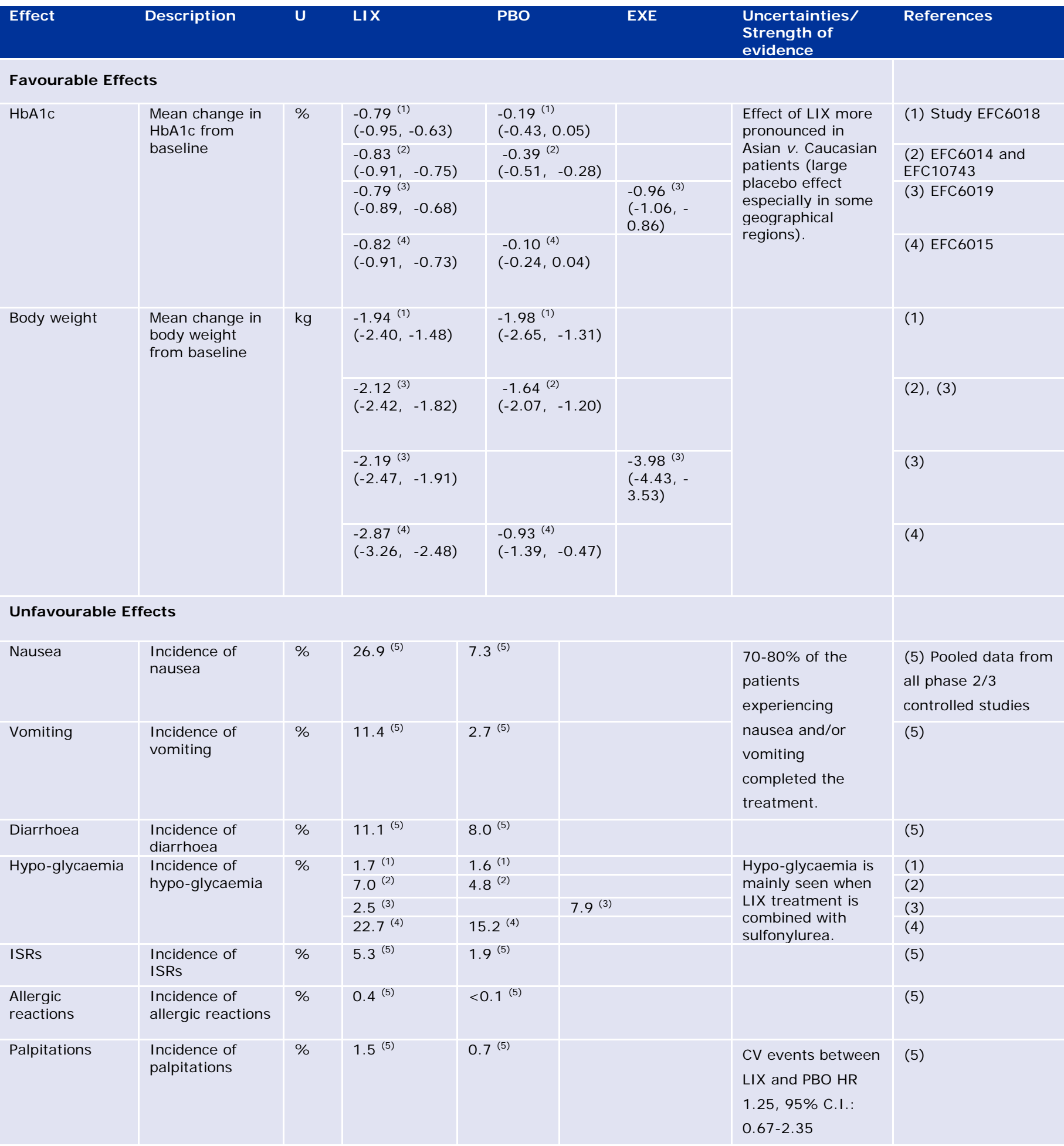

Abbreviations:

U: unit; LIX: lixisenatide; PBO: placebo; EXE: exenatide; HbAlc: glycosylated haemoglobin; ISRs: injection site reactions. 
Table 2. Quantitative frameworks: Commonly held views in favour and against

Against

Require more effort than the implicit holistic approach.

Require additional training.

They do not reflect the mental processes, which are prevalently intuitive.

They are highly subjective.

They give a false sense of precision.

They are a "black box" that may obscure the expert judgment.

High precision is unattainable in the regulatory setting.

They oversimplify the complexity of the data structure and decision context into a "single number".

The benefit-risk balance is often self-evident from the data. Complex quantitative frameworks are unnecessary.

Whose values? The authority of the decisionmakers will be questioned.

\section{In favour}

Some extra effort is needed, at least initially, to structure the model. Updating of models is very efficient.

Minimal training necessary.

Intuition can lead to error and bias, particularly for inexperienced assessors.

Implicit mental processes are difficult to communicate.

No more subjective than any other decisionmaking strategy; but the subjectivity is handled explicitly.

Weights and outputs from the model have to be considered critically, alongside uncertainties.

The methods are relatively straightforward and can be easily understood.

The structure of the model (options, outcomes, consequences, objectives and criteria) and all quantitative inputs (utilities, probabilities and criterion weights) are made explicit.

Uncertainty can be managed explicitly by exploring different weights and assumptions.

The methods are used to explore the interaction of many components in the decision.

A single number summary is an abuse of the model.

Results can be characterized probabilistically with the contributions of different components displayed.

No need to use in self-evident cases. Still, decision-making may not be self-evident to all stakeholders.

The impact of different inputs (e.g., from patients) can be explored.

Regulator's decisions can be scrutinised. 
Table 3. Steps in the BRAT Framework

\section{Step Description}

1. Define the decision context

2. Identify outcomes

3. Identify and extract source data

4. Customize the framework

5. Assess outcome importance

6. Display and interpret key benefit-risk metrics
Define drug, dose, formulation, indication, patient population, comparator(s), time horizon for outcomes, perspective of the decisionmakers (regulator, sponsor, patient, or physician).

Select all important outcomes and create the initial value tree. Define a preliminary set of outcome measures/endpoints for each. Document rationale for outcomes included/excluded.

Determine and document all data sources (e.g., clinical trials, observational studies).

Extract all relevant data into the data source table, including detailed references and any annotations to help the subsequent interpretations.

Modify the value tree based on further review of the data and clinical expertise. Refine the outcome measures/endpoints. May include tuning of outcomes not considered relevant to a particular benefit-risk assessment or that vary in relevance by stakeholder groups.

Apply or assess any ranking or weighting of outcome importance to decision-makers or other stakeholders.

Summarize source data into tabular and graphical displays to aid interpretation.

Challenge summary metrics, review source data, identify and fill any information gaps.

Interpret summary information.

Table 4. Examples of important behaviours and decisions by consumer when using a nonprescription drug which impact benefit-risk. The consumer will make these decisions based on the drug's label, potentially augmented by a pharmacist, as well as their own knowledge and self-assessment of their condition. See the World Self-Medication Industry tools for the Benefit-Risk Assessment of Nonprescription Drugs (BRAND tools)

Does the consumer's current clinical condition match the indication for the drug?

Does the consumer have any contraindications for use of the drug?

Does the consumer have any symptoms or other medical history which require consultation with a physician prior to using the drug?

Is the consumer taking any other drugs for which there is a drug interaction warning with the drug?

Will the consumer take the drug at the proper dose, at the correct time interval and for the appropriate duration of treatment?

Will the consumer discontinue use of the drug if adverse effects develop?

Will the consumer seek medical attention if their clinical condition evolves so that self-management is no longer appropriate? 